\title{
A sufficient condition for the absence of the sign problem in the fermionic quantum Monte-Carlo algorithm
}

\author{
Congjun Wu and Shou-Cheng Zhang \\ Department of Physics, McCullough Building, Stanford University, Stanford CA 94305-4045
}

\begin{abstract}
Quantum Monte-Carlo (QMC) simulations involving fermions have the notorious sign problem. Some well-known exceptions of the auxiliary field QMC algorithm rely on the factorizibility of the fermion determinant. Recently, a fermionic QMC algorithm 1] has been found in which the fermion determinant may not necessarily factorizable, but can instead be expressed as a product of complex conjugate pairs of eigenvalues, thus eliminating the sign problem for a much wider class of models. In this paper, we present general conditions for the applicability of this algorithm and point out that it is deeply related to the time reversal symmetry of the fermion matrix. We apply this method to various models of strongly correlated systems at all doping levels and lattice geometries, and show that many novel phases can be simulated without the sign problem.

PACS numbers: 02.70.Ss, 71.10.Fd
\end{abstract}

\section{INTRODUCTION}

Understanding the physics of strongly correlated many body systems is a main focus of condensed matter physics today. However, most models with strong interactions can not be solved exactly except in one dimension. Presently, there are no systematic non-perturbative analytic methods which work in higher dimensions. Largely because of this reason, numerical simulations such as exact diagonalization (ED), density-matrix renormalization group (DMRG), quantum Monte-Carlo (QMC) are extensively performed to study strongly correlated systems. However, each of the numerical methods has its own limitations. The ED can only be performed in a very small sample size, and the DMRG method is largely restricted to one-dimensional systems. In contrast, QMC simulation is the only systematic and scalable method with sufficient numerical accuracy for higher dimensional problems. However, QMC also has the notorious fermion sign problem which makes low temperature properties inaccessible.

In lattice systems, a particular version of QMC uses the auxiliary field method introduced by Blankenbecler, Scalapino and Sugar [2], with fruitful results. Because one can not directly sample the fermionic Grassmann fields, the standard process is to perform a HubbardStratonovich (HS) transformation to decouple the fourfermion interaction terms, and then to integrate out the fermions [2]. The resulting fermion functional determinant works as the statistical weight for sampling the auxiliary fields. However, generally speaking the fermion determinant may not be positive, and can even be complex in some cases. The sign or the phase of the fermion determinants can lead to dramatic cancellations which makes statistical errors to scale exponentially as the inverse of the temperature and size of the system. This notorious sign problem is the major obstacle in applying QMC to fermionic systems. A successful solution to the sign problem would obviously lead to great advances in quantum many body physics.
There are a few exceptions where the sign problem is absent, such as the negative $U$ Hubbard model and the positive $U$ Hubbard model in a bipartite lattice at the half-filling [3]. In both cases, the fermion determinant after the HS decomposition can be factorized into to two real parts with the same sign. It is therefore positive-definite. Unfortunately, general fermion determinants may not be factorizable for more complicated models and the majority of models do have the sign problem. In recent years, several other algorithms have been proposed which partially solves the minus sign problem [4, 5, 6, 6, 8].

Recently, it has been shown that the minus sign problem can be eliminated without relying on the factorizibility of the fermion determinant, therefore, a broader class of models can be simulated by the QMC algorithm 1]. The fermion determinant can always be expressed as a product of its eigenvalues; under certain conditions, the eigenvalues of the fermion determinant always appear in complex conjugate pairs, thus making the fermion determinant positive definite. In this article, we shall show that the property of conjugate eigenvalue pairs follows from the time reversal symmetry of the HS decoupled Hamiltonian, and can be viewed as a generalization of the Kramers theorem in quantum mechanics. We shall call this method the $T$-invariant decomposition (time reversal invariant decomposition). This method does not lead to any improvement for the single band Hubbard model, but significantly extends the applicability of the QMC to multi-band, multi-layer or higher spin models. This algorithm is particularly useful for Hubbard models with higher spins, which can be accurately realized in systems of cold atoms. Recently, Assaad et al. [9] applied the QMC to generalized Hubbard models with more bands. Imposing the factorizibility condition of the fermion determinant, they found that they could extend the parameter regime for QMC free of the sign problem only by scarifying the spin rotational invariance. However, applying our method of $T$-invariant decomposition without requiring factorizibility, we shall show that multi- 
band or higher spin Hubbard models can be simulated for an extended parameter regime without scarifying the spin rotational invariance. This QMC algorithm based on $T$-invariant decomposition has been recently applied to conclusively demonstrate the staggered current carrying ground state in a bi-layer model [10].

The rest of this article is outlined as follows: In Sec. III the sign problem for the spin $1 / 2$ Hubbard model is reviewed. In Sec. III we prove the fundamental theorem of $T$-invariant decomposition and show the absence of the sign problem. In Sec. IV we employ the algorithm to the spin $3 / 2$ Hubbard model and the generalized arbitrary spin $n-1 / 2$ fermionic Hubbard model. In Sec. D. we apply it to a bi-layer model introduced by Scalapino, Zhang and Hanke 11], which can be mapped into the spin 3/2 Hubbard model. In Sec. VI we discuss the algorithm in the model Hamiltonians with bond interactions and various exotic phases. Final conclusions are presented in Sec. VII

\section{THE SIGN PROBLEM IN THE SPIN $1 / 2$ HUBBARD MODEL}

In this section, we review the sign problem in the spin $1 / 2$ Hubbard model and interpret its absence in the negative $U$ case as due to its time reversal properties of the
HS decomposition. The Hubbard model on the lattice is commonly defined as

$$
\begin{aligned}
H & =-t \sum_{i j, \sigma}\left(c_{i \sigma}^{\dagger} c_{j \sigma}+h . c .\right)-\mu \sum_{i} n(i) \\
& +U \sum_{i}\left(n_{\uparrow}(i)-\frac{1}{2}\right)\left(n_{\downarrow}(i)-\frac{1}{2}\right),
\end{aligned}
$$

with $t$ the hopping integral, $\mu$ the chemical potential, $\sigma=\uparrow, \downarrow, n_{\sigma}(i)=c_{i \sigma}^{\dagger} c_{i \sigma}$ and $n(i)=n_{\uparrow}(i)+n_{\downarrow}(i)$. At half-filling and on a bipartite lattice, the particle-hole symmetry ensures that $\mu=0$.

To perform the QMC simulation, we first need to decouple the 4-fermion interaction terms using the HS transformations by the Gaussian integral:

$$
\begin{aligned}
\exp \left(\frac{1}{2} A^{2}\right) & =\sqrt{2 \pi} \int d x \exp \left(-\frac{1}{2} x^{2}-x A\right) \\
\exp \left(-\frac{1}{2} A^{2}\right) & =\sqrt{2 \pi} \int d x \exp \left(-\frac{1}{2} x^{2}-i x A\right) .
\end{aligned}
$$

Various HS decoupling schemes are discussed in Ref. [12]. For $U<0$, it is convenient to decouple Eq. (11) in the density channel and then integrate out the fermions. The resulting partition function is given by

$$
\begin{aligned}
Z & =\int D c^{\dagger} D c \exp \left\{-\int_{0}^{\beta} d \tau\left(c_{\sigma}^{\dagger} \frac{\partial}{\partial \tau} c_{\sigma}+H\right)\right\} \\
& =\int D n D c^{\dagger} D c \exp \left\{-\frac{|U|}{2} \int_{0}^{\beta} d \tau \sum_{i}(n(i, \tau)-1)^{2}\right\} \exp \left\{-\int_{0}^{\beta} d \tau\left(H_{K}+H_{I}(\tau)\right)\right\} \\
& =\int D n \exp \left\{-\frac{|U|}{2} \int_{0}^{\beta} d \tau \sum_{i}(n(i, \tau)-1)^{2}\right\} \operatorname{det}\{I+B\}
\end{aligned}
$$

where $n(i, \tau)$ is a real HS bose density field. The imaginary-time independent kinetic energy term $H_{K}$ and the imaginary-time dependent decoupled interaction term $H_{I}(\tau)$ can be expressed as

$$
\begin{aligned}
H_{K} & =\sum_{i j} c_{i \sigma}^{\dagger} h_{i j, \sigma \sigma^{\prime}}^{K} c_{j \sigma^{\prime}}, \quad H_{I}=\sum_{i} c_{i \sigma}^{\dagger} h_{i j, \sigma \sigma^{\prime}}^{I} c_{j \sigma^{\prime}}, \\
h_{i j, \sigma \sigma^{\prime}}^{K} & =\left\{-t\left(\delta_{i, j+\hat{x}}+\delta_{i, j-\hat{x}}+\delta_{i, j+\hat{y}}+\delta_{i, j-\hat{y}}\right)-\mu \delta_{i j}\right\} \delta_{\sigma \sigma^{\prime}} \\
h_{i j, \sigma \sigma^{\prime}}^{I} & =U n(i, \tau) \delta_{i j} \delta_{\sigma \sigma^{\prime}} .
\end{aligned}
$$

Note that the matrix kernels $h_{i j, \sigma \sigma^{\prime}}^{K}$ and $h_{i j, \sigma \sigma^{\prime}}^{I}$ entering in Eq. (5), as well as the $I+B$ matrix itself, are $2 N \times 2 N$ matrices, if the lattice system under simulation has $N=$ $L_{x} \times L_{y}$ sites. In the subsequent discussions, we shall simply use the second quantized operators $H_{K}$ and $H_{I}$ interchangeably with the first quantized matrix kernels $h_{K}$ and $h_{I}$ to save some writing, whenever their meanings are obvious from the context.

In practice, $I+B$ needs to discretized as

$$
\begin{aligned}
& I+B=I+e^{-\Delta \tau H_{K}} e^{-\Delta \tau H_{i}\left(\tau_{l}\right)} e^{-\Delta \tau H_{K}} e^{-\Delta \tau H_{i}\left(\tau_{l-1}\right)} \ldots \\
& \ldots e^{-\Delta \tau H_{K}} e^{-\Delta \tau H_{i}\left(\tau_{1}\right)}
\end{aligned}
$$

where $\Delta \tau=\beta / l$ is the discretized time slice.

Similarly, at $U>0$, Eq. (11) can be decomposed in the 
spin density channel as

$$
Z=\int D S_{z} \exp \left\{-2 U \int_{0}^{\beta} d \tau \sum_{i} S_{z}^{2}(i, \tau)\right\} \operatorname{det}\{I+B\}
$$

with the same expression for $B$ as in Eq. (5), but with $H_{I}$ replaced by

$$
H_{I}(\tau)=-2 U \sum_{i}\left\{c_{i \alpha}^{\dagger}(\tau) \sigma_{\alpha \beta}^{z} c_{i \beta}(\tau)\right\} S_{z}(i, \tau) .
$$

It is well known that the spin $1 / 2$ Hubbard model is free of the sign problem either for $U<0$ or for $U>$ 0 at half-filling and in a bipartite lattice 3,12 . The usual proof is based on the factorization of the fermion determinant as

$$
\operatorname{det}\{I+B\}=\operatorname{det}\left\{I+B_{\uparrow}\right\} \operatorname{det}\left\{I+B_{\downarrow}\right\} .
$$

In the negative $U$ case, the HS decomposition in Eq. (4) enables such a factorization, and $B_{\uparrow}$ is identical to $B_{\downarrow}$ for any HS field configurations. Therefore $\operatorname{det}\{I+B\}$ is the square of a real number and thus positive definite. Generally speaking, in the positive $U$ case, the HS decomposition in Eq. (8) still enables factorization, but $\operatorname{det}\left\{I+B_{\uparrow}\right\}$ is different from $\operatorname{det}\left\{I+B_{\downarrow}\right\}$, thus the sign problem appears. However, at half-filling and on a bipartite lattice, it is possible to change the sign of $U$ while keeping the kinetic energy part invariant by a partial particle-hole transformation only on spin down particles

$$
c_{i \uparrow} \rightarrow c_{i \uparrow}, \quad c_{i \downarrow} \rightarrow(-)^{i} c_{i \downarrow}^{\dagger},
$$

then the above algorithm is also applicable. Nevertheless, this transformation can not be applied to lattices which are not bipartite, or away from the half-filling $(\mu \neq 0)$, thus the sign problem remains in general.

Recently, an anisotropic two band model explicitly breaking the spin rotational symmetry is also shown to be free of the sign problem [9]. The Hamiltonian is defined by

$$
\begin{aligned}
H & =-t \sum_{i j, \sigma}\left(c_{i \sigma}^{\dagger} c_{j \sigma}+h . c .\right)-\mu \sum_{i, \sigma} n_{\sigma}(i) \\
& -|U| \sum_{i}\left(n_{1}(i)-n_{2}(i)+n_{3}(i)-n_{4}(i)\right)^{2}
\end{aligned}
$$

where $n_{\sigma}(i)=c_{\sigma}^{\dagger}(i) c_{\sigma}(i)$ are particle densities for each spin components $\sigma=1,2,3,4$. The interaction part can be decoupled as

$$
\begin{aligned}
Z & =\int D S \exp \left\{-|U| \int_{0}^{\beta} d \tau \sum_{i} S^{2}(i, \tau)\right\} \\
& \times \exp \left\{-\int_{0}^{\beta} d \tau\left(H_{0}+H_{I}(\tau)\right)\right\} \\
H_{I}(\tau) & =\sum_{i}\left(c_{i, 1}^{\dagger} c_{i, 1}-c_{i, 2}^{\dagger} c_{i, 2}+c_{i, 3}^{\dagger} c_{i, 3}-c_{i, 4}^{\dagger} c_{i, 4}\right) S(i, \tau)
\end{aligned}
$$

This HS decomposition enables the factorization of the fermion determinant as

$$
\operatorname{det}\{I+B\}=\operatorname{det}\{I+B\}_{12} \operatorname{det}\{I+B\}_{34},
$$

where $\operatorname{det}\{I+B\}_{12}$ and $\operatorname{det}\{I+B\}_{34}$ for spin components 1,2 and 3,4 respectively are identical and real. Therefore, the fermion determinant is positive in this case as well. However, a disadvantage of this model is the explicit breaking of the spin rotational symmetry.

\section{FUNDAMENTAL THEOREM OF $T$-INVARIANT DECOMPOSITION}

We now show that the condition of factorizibility of the fermion determinant is unnecessarily restrictive, and a more general condition can be precisely stated. The fermion determinant is a product of all the eigenvalues. Since $I+B$ involves a time ordered product, it may not be Hermitian and the eigenvalues may be complex in general. Because the ensemble of HS field configurations is arbitrary, one would naively not expect any special relations among the eigenvalues. Surprisingly, the time reversal symmetry provides an important relationship among the eigenvalues. To formulate the fundamental theorem, we consider $H_{K}$ and $H_{I}$ in the $I+B$ matrix of Eq. (6) to be the HS decomposed single particle Hamiltonian matrix derived from a general Hamiltonian, not necessarily the $s=1 / 2$ Hubbard model.

Theorem: If there exists an anti-unitary operator $T$, such that

$$
T H_{K} T^{-1}=H_{K}, \quad T H_{I} T^{-1}=H_{I}, \quad T^{2}=-1,
$$

then the eigenvalues of the $I+B$ matrix always appear in complex conjugate pairs, i.e., if $\lambda_{i}$ is an eigenvalue, then $\lambda_{i}^{*}$ is also an eigenvalue. If $\lambda_{i}$ is real, it is twofold degenerate. In this case, the fermion determinant is positive definite,

$$
\operatorname{det}(I+B)=\prod_{i}\left|\lambda_{i}\right|^{2} \geq 0
$$

Proof: From the condition of the theorem stated in Eq. (13), it obviously follows that $T(I+B) T^{-1}=(I+B)$. For simplicity, we first consider the case where $I+B$ is an $n \times n$ dimensional diagonalizable matrix, i.e., there exists a non-singular matrix $P$ satisfying

$$
P^{-1}(I+B) P=\operatorname{diag}\left\{\lambda_{1}, \lambda_{2}, \ldots, \lambda_{n}\right\} .
$$

The $n$ columns of $P$ can be viewed as a set of linearly independent state-vectors

$$
P=\left\{\left|\Psi_{1}\right\rangle,\left|\Psi_{2}\right\rangle, \ldots,\left|\Psi_{n}\right\rangle\right\}
$$

Suppose that $\left|\Psi_{i}\right\rangle$ is an eigenvector with eigenvalue $\lambda_{i}$, i.e. $(I+B)\left|\Psi_{i}\right\rangle=\lambda_{i}\left|\Psi_{i}\right\rangle$. Using the anti-unitary property of $T$, we see that

$$
(I+B) T\left|\Psi_{i}\right\rangle=T(I+B) T^{-1} T\left|\Psi_{i}\right\rangle=\lambda_{i}^{*} T\left|\Psi_{i}\right\rangle .
$$


Therefore, $T\left|\Psi_{i}\right\rangle$ is also an eigenvector, with eigenvalue $\lambda_{i}^{*}$. Since $T^{2}=-1, T\left|\Psi_{i}\right\rangle$ and $\left|\Psi_{i}\right\rangle$ are orthogonal to each other. This shows that $\lambda_{i}$ and $\lambda_{i}^{*}$ are two different eigenvalues, thus the eigenvalues of $I+B$ appear in complex conjugate pairs as stated in the theorem. If $I+B$ is Hermitian, our theorem reduces to Kramer's theorem on the time reversal symmetry in quantum mechanics, stating that the eigenvalues of $I+B$ are real, and two-fold degenerate.

In the general case, $I+B$ may not be diagonalizable, instead it can always be transformed into the Jordan normal form as diagonal blocks

$$
P^{-1}(I+B) P=\operatorname{diag}\left\{J_{1}, J_{2}, \ldots, J_{k}\right\},
$$

where $P$ is an $n \times n$ non-singular matrix as before and $J_{i}$ is an $l_{i} \times l_{i}$ bi-diagonal matrix as

$$
J_{i}=\left(\begin{array}{ccccc}
\lambda_{i} & 1 & & & \\
& \cdot & \cdot & & \\
& & \cdot & \cdot & \\
& & \lambda_{i} & 1 \\
& & & \lambda_{i}
\end{array}\right)
$$

The determinant of $I+B$ is still the product of all the eigenvalues

$$
\operatorname{det}(I+B)=\prod_{i=1}^{k}\left(\lambda_{i}\right)^{l_{i}}
$$

As in Eq. 16. $P$ can be viewed as $n$ linearly independent column state-vectors as

$$
P=\left\{P_{1}, P_{2}, \ldots, P_{k}\right\},
$$

where each $P_{i}$ is an $n \times l_{i}$ matrix containing $l_{i}$ column state-vectors

$$
P_{i}=\left\{\left|\Psi_{m+1}\right\rangle, \ldots,\left|\Psi_{m+l_{i}}\right\rangle\right\}, \quad m=\sum_{j=1}^{i-1} l_{j}
$$

For each Jordan block $J_{i}$, it satisfies

$$
(I+B) P_{i}=P_{i} J_{i}
$$

thus among the $l_{i}$ state-vectors in $P_{i},\left|\Psi_{m+1}\right\rangle$ is the only eigenvector with eigenvalule $\lambda_{i}$. It is straightforward to show that

$$
(I+B)\left(T P_{i}\right)=\left(T P_{i}\right) J_{i}^{*},
$$

where $\left(T P_{i}\right)$ is defined as

$$
\left(T P_{i}\right)=\left\{T\left|\Psi_{m+1}\right\rangle, \ldots, T\left|\Psi_{m+l_{i}}\right\rangle\right\},
$$

and $T\left|\Psi_{m+1}\right\rangle$ is the only eigenvector with eigenvalue $\lambda_{i}^{*}$ in $\left(T P_{i}\right)$. Again since $\left|\Psi_{m+1}\right\rangle$ and $T\left|\Psi_{m+1}\right\rangle$ are orthogonal to each other, $\left(T P_{i}\right)$ contains different state vectors from what $P_{i}$ does. As a result, $J_{i}$ and $J_{i}^{*}$ are different Jordan blocks. As before, the Jordan blocks appear in

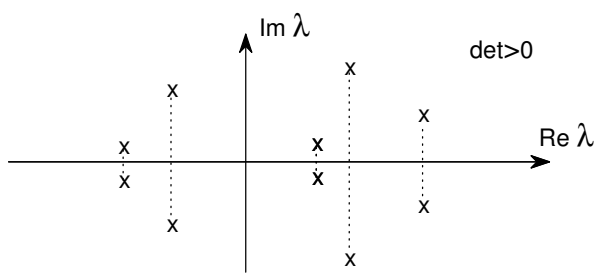

(a)

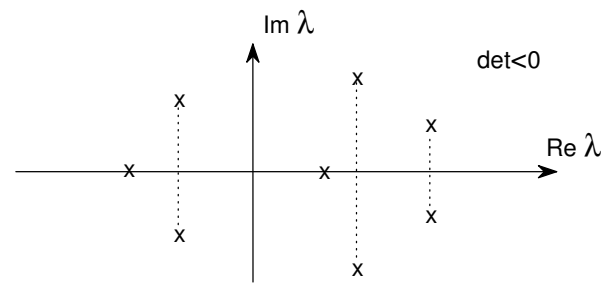

(b)

FIG. 1: Distribution of eigenvalues in the complex plane. (a) Eigenvalues of a fermion matrix satisfying the conditions of our theorem are always paired. (b) Complex eigenvalues of a generic real matrix are paired, but real eigenvalues are not two-fold degenerate in general, leading to negative determinants.

complex conjugate pairs and so do the eigenvalues. This completes the proof for the general case of $I+B$.

Since the anti-unitary operator $T$ used in our theorem shares similar properties as the time reversal transformation in quantum mechanics, we call our method $T$-invariant decomposition. However, it is important to emphasize that any anti-unitary operator with the stated mathematical properties could work here. In some examples we shall discuss, $T$ does not have the explicit physical meaning of the time reversal transformation.

It is also important to point out that the $T^{2}=-1$ condition is essential for our theorem. In the case when the fermion matrix is real, one can define a trivial antiunitary operator $T=C$, where $C$ denotes the complex conjugation. In this case, if the eigenvalue $\lambda_{i}$ is complex, i.e., $\lambda_{i} \neq \lambda_{i}^{*}$, then $\lambda_{i}^{*}$ must also be an eigenvalue. However, when $\lambda_{i}$ is real, it is in general not two-fold degenerate, since $|\Psi\rangle$ and $T|\Psi\rangle$ may not be orthogonal for the case of $T^{2}=1$. In this case, an odd number of negative eigenvalues would lead to a negative determinant. The distribution of eigenvalues in the complex plane for a fermion matrix satisfying the condition of our theorem and the eigenvalues of a generic real fermion matrix is illustrated in Fig. 1] When the conditions of our theorem is violated, either the complex conjugate eigenvalue pairs collide on the real axis and move off from each other along the real axis, or the two-fold degenerate eigenvalues move off directly from each other along the real axis.

A restricted version of our theorem was originally discussed in the context of nuclear physics $[5]$. However, 
these authors overlooked the case that $I+B$ may not be diagonalizable, thus their proof was not complete. In addition, our $T$-transformation is not restricted to the physical time-reversal transformation as in Ref. [5], thus the theorem applies to a much wider class of models.

We now illustrate this general theorem for the case of the $s=1 / 2$ Hubbard model. For the spin $1 / 2$ system on each site, the time-reversal transformation $T$ is defined as $T(i)=R(i) C$, satisfying $T^{2}(i)=-1$, where

$$
R=-i \sigma_{y}=\left(\begin{array}{cc}
0 & -1 \\
1 & 0
\end{array}\right) .
$$

For the entire system, the time-reversal operator is defined as the direct product $T=\left(\Pi_{i} \otimes R(i)\right) C$. The four independent fermion bilinears in the particle-hole channel can be classified as the particle number $n(i)=\psi_{i, \alpha}^{\dagger} \psi_{i, \alpha}$ and spin $\vec{S}(i)=\psi_{i, \alpha}^{\dagger}(\sigma / 2)_{\alpha \beta} \psi_{i, \beta}$, which are even and odd under the $T$ transformation respectively:

$$
T n(i) T^{-1}=n(i), \quad T \vec{S}(i) T^{-1}=-\vec{S}(i) .
$$

Now we can understand the absence of sign problem in the negative $U$ case as follows. The density channel decomposition is $T$-invariant, namely, $T\left(H_{K}+\right.$ $\left.H_{I}(\tau)\right) T^{-1}=H_{K}+H_{I}(\tau)$. The conditions of our theorem is satisfied and the fermion determinant is thus positive. For $U>0$, the Hamiltonian can be decoupled in the density channel at the cost of involving the imaginary number $i$, or decoupled in the spin channel with only real numbers. In either cases, while $H_{K}$ is still even under $T$, $H_{I}$ is odd. The conditions of our theorem does not apply, and the sign problem appears in general.

For a general interacting fermion model, we can always express $T=R C$, where $R R^{*}=-1$ and $R^{*}$ is the complex conjugate of $R$. In many cases, $R$ is purely real, and it reduces to $R^{2}=-1$. The general condition for our theorem then reads

$$
R\left(H_{K}+H_{I}\right) R^{-1}=\left(H_{K}+H_{I}\right)^{*},
$$

with the unitary matrix $R$ satisfying $R R^{*}=-1$ for any configurations of the HS field. Again we emphasis that the precise form for $R$ in Eq. (26) is not necessary.

While our new method does not lead to any improvement of the sign problem for the $s=1 / 2$ Hubbard model, we shall show now that it significantly improves the QMC algorithm for multi-band, multi-layer and higher spin models, since the conditions for our theorem is far less restrictive than the condition for the factorizibility of the fermion determinant. Let us illustrate the general idea here by looking at the example of a two band spin $1 / 2$ model or a spin $3 / 2$ model. In this case, we have fermion operators $\psi_{i, \beta}$ within one unit cell, where $\beta=1,2,3,4$. Therefore, there are 16 fermion bilinears, of the form $M^{I}=\psi_{i, \alpha}^{\dagger} M_{\alpha \beta}^{I} \psi_{i, \beta}$, where $I=1, \ldots, 16$. The $16 M_{\alpha \beta}^{I}$ matrices can in general be expressed in a complete basis in terms of the product of $s=3 / 2$ matrices $S_{i}$ :

$I$

$$
\begin{aligned}
& S^{i}, \quad i=1,2,3, \\
& \xi_{i j}^{a} S_{i} S_{j}, \quad a=1, . ., 5, \quad \xi_{i j}^{a}=\xi_{j i}^{a}, \quad \xi_{i i}^{a}=0, \\
& \xi_{i j k}^{L} S_{i} S_{j} S_{k}, \quad L=1, . ., 7, \quad \xi_{i j k}^{L}=\xi_{j i k}^{L}, \quad \xi_{i i k}^{L}=0,
\end{aligned}
$$

where $\xi$ 's are fully symmetric, traceless tensors. If one insists on the factorizibility of the fermion determinant, one could only perform the HS decomposition in the density channel using the identity matrix $I$. However, since the $\xi_{i j}^{a} S_{i} S_{j}$ matrix contains an even power of spin matrices, it is also even under time reversal. HS decomposition in this channel does not lead to factorization of the fermion determinant, but according to our general theorem, it does lead to paired eigenvalues, and therefore, a positive fermion determinant. As we see from this non-trivial example, our method of $T$-invariant decomposition is indeed more general and more powerful compared with the traditional method of factorization. We shall show the enlarged parameter space for QMC algorithm explicitly in the next section.

\section{APPLICATION IN SPIN 3/2 AND $n-1 / 2$ HUBBARD MODEL}

In this section, we apply the method of $T$-invariant decomposition to the $s=\frac{3}{2}$ model as an explicit example, and discuss the sign problem accordingly. After that, we generalize it to arbitrary fermionic Hubbard models with $s=n-1 / 2$. These models are not of only academic interests. In fact, the rapid progress in ultra-cold atomic systems provides an opportunity to study higher spin fermions. The simplest cases are the spin $3 / 2$ atoms, such as ${ }^{9} \mathrm{Be},{ }^{132} \mathrm{Cs},{ }^{135} \mathrm{Ba},{ }^{137} \mathrm{Ba}$ atoms. Another important research direction is the trapped atoms in an optical lattice, formed by the standing wave laser beams, where the Hubbard model is a good approximation for these neutral atoms.

\section{A. The $s=3 / 2$ Hubbard model}

The spin $3 / 2$ Hubbard model is defined as 1 ]

$$
\begin{aligned}
& H=-t \sum_{\langle i j\rangle, \sigma}\left\{c_{i \sigma}^{\dagger} c_{j \sigma}+h . c .\right\}-\left(\mu+\mu_{0}\right) \sum_{i \sigma} c_{i \sigma}^{\dagger} c_{i \sigma} \\
& +U_{0} \sum_{i} P_{0}^{\dagger}(i) P_{0}(i)+U_{2} \sum_{i, m= \pm 2, \pm 1,0} P_{2 m}^{\dagger}(i) P_{2 m}(i),
\end{aligned}
$$

with $\mu_{0}=\left(U_{0}+5 U_{2}\right) / 4$. $\mu$ is fixed to be zero at halffilling on a bipartite lattice, to ensure the particle-hole (p-h) symmetry generated by the transformation $c_{i, \sigma} \rightarrow$ $(-)^{i} c_{i, \sigma}^{\dagger}$. Because of the Pauli's exclusion principle, only on-site interactions in the total spin singlet $\left(S_{T}=0\right)$ and the quintet $\left(S_{T}=2\right)$ channels are allowed. $P_{0}^{\dagger}, P_{2 m}^{\dagger}$ are 
the singlet and quintet pairing operators defined by

$$
\begin{aligned}
& P_{0}^{\dagger}(i)\left(P_{20}^{\dagger}(i)\right)=\frac{1}{\sqrt{2}}\left\{c_{i, \frac{3}{2}}^{\dagger} c_{i,-\frac{3}{2}}^{\dagger} \mp c_{i, \frac{1}{2}}^{\dagger} c_{i,-\frac{1}{2}}^{\dagger}\right\}, \\
& P_{2,2}^{\dagger}(i)=c_{i, \frac{3}{2}}^{\dagger} c_{i, \frac{1}{2}}^{\dagger}, \quad P_{2,1}^{\dagger}(i)=c_{i, \frac{3}{2}}^{\dagger} c_{i,-\frac{1}{2}}^{\dagger}, \\
& P_{2,-1}^{\dagger}(i)=c_{i, \frac{1}{2}}^{\dagger} c_{i,-\frac{3}{2}}^{\dagger}, P_{2,-2}^{\dagger}(i)=c_{i,-\frac{1}{2}}^{\dagger} c_{i,-\frac{3}{2}}^{\dagger} .
\end{aligned}
$$

The $s=3 / 2$ Hubbard model has an exact $S O(5)$ or equivalently, $S p(4)$ symmetry, without any fine tuning of the parameters 1]. This follows from the fact that singlet and quintet channel interactions can also be interpreted as $S O(5)$ group's singlet and 5-vector representations. When $U_{0}=U_{2}$, the model has a larger symmetry, namely the $S U(4)$ symmetry. The $S U(4)$ symmetric Hubbard model has been extensively studied in the transition metal oxides with double orbital degeneracy 13 .

To illustrate the $T$-invariant decomposition for this model, we first define the 4-component spinor

$$
\psi(i)=\left(c_{\frac{3}{2}}(i), c_{\frac{1}{2}}(i), c_{-\frac{1}{2}}(i), c_{-\frac{3}{2}}(i)\right)^{T} .
$$

In this representation, we define five $4 \times 4$ Dirac $\Gamma^{a}(1 \leq$ $a \leq 5)$ matrices to construct the $S p(4)$ or $S O(5)$ algebra as

$\Gamma^{1}=\left(\begin{array}{cc}0 & i I \\ -i I & 0\end{array}\right), \Gamma^{2,3,4}=\left(\begin{array}{cc}\vec{\sigma} & 0 \\ 0 & -\vec{\sigma}\end{array}\right), \Gamma^{5}=\left(\begin{array}{cc}0 & -I \\ -I & 0\end{array}\right)$,

where $I$ and $\vec{\sigma}$ are the $2 \times 2$ unit and Pauli matrices. The ten $S O(5)$ generators are defined as $\Gamma^{a b}=$ $-\frac{i}{2}\left[\Gamma^{a}, \Gamma^{b}\right](1 \leq a, b \leq 5)$. Since the $S O(5)$ group is equivalent to the $S p(4)$ group, there exists a symplectic matrix $R$, with the properties 11

$$
\begin{aligned}
& R^{2}=-1, \quad R^{\dagger}=R^{-1}={ }^{t} R=-R \\
& R \Gamma^{a} R^{-1}={ }^{t} \Gamma^{a}, \quad R \Gamma^{a b} R^{-1}=-{ }^{t} \Gamma^{a b} .
\end{aligned}
$$

In our explicit representation,

$$
R=\Gamma^{1} \Gamma^{3}=\left(\begin{array}{cc}
0 & -i \sigma_{2} \\
-i \sigma_{2} & 0
\end{array}\right) .
$$

Using the $R$ matrix, the $s=3 / 2$ Hubbard interaction can be written in an explicitly $S O(5)$ symmetric fashion as

$$
\begin{aligned}
H & =-t \sum_{i j}\left(\psi^{\dagger}(i) \psi(j)+\text { h.c. }\right)-\left(\mu+\mu_{0}\right) \sum_{i} \psi^{\dagger}(i) \psi(i) \\
& +\frac{U_{0}}{2} \sum_{i} \eta^{\dagger}(i) \eta(i)+\frac{U_{2}}{2} \sum_{i, a} \chi^{\dagger, a}(i) \chi^{a}(i)
\end{aligned}
$$

where $\eta^{\dagger}(i)=\psi^{\dagger}(i)(R / 2) \psi^{\dagger}(i)$ is the singlet paring operator, and $\chi^{a, \dagger}(i)=\psi^{\dagger}(i)\left(\Gamma^{a} R / 2\right) \psi^{\dagger}(i)$ are the polar forms of the quintet paring operators in Eq. (31).

In order to implement the method of $T$ invariant decomposition, we first need to express the interaction terms in the particle-hole channel, rather than the particle-particle channel. In the particle-hole channel, there are 16 bilinear fermionic operators, which can be classified into the scalar, vector, and anti-symmetric tensors (generators) of the $S O(5)$ group as

$$
\begin{aligned}
n(i) & =\psi_{\alpha}^{\dagger}(i) \psi_{\alpha}(i) \\
n_{a}(i) & =\frac{1}{2} \psi_{\alpha}^{\dagger}(i) \Gamma_{\alpha \beta}^{a} \psi_{\beta}(i), \quad 1 \leq a \leq 5 \\
L_{a b}(i) & =-\frac{1}{2} \psi_{\alpha}^{\dagger}(i) \Gamma_{\alpha \beta}^{a b} \psi_{\beta}(i), \quad 1 \leq a<b \leq 5,
\end{aligned}
$$

where $n(i)$ is the particle number operator, $n_{a}(i)$ and $L_{a b}(i)$ represent the spin degrees of freedom. The ten $S O(5)$ generators are often conveniently denoted as

$$
L_{a b}(i)=\left(\begin{array}{ccccc}
0 & \operatorname{Re} \pi_{\mathrm{x}} & \operatorname{Re} \pi_{\mathrm{y}} & \operatorname{Re} \pi_{\mathrm{z}} & Q \\
& 0 & -S_{z} & S_{y} & \operatorname{Im} \pi_{\mathrm{x}} \\
& & 0 & -S_{x} & \operatorname{Im} \pi_{\mathrm{y}} \\
& & & 0 & \operatorname{Im} \pi_{\mathrm{z}}
\end{array}\right) .
$$

Although the similar symbols are used in the $S O(5)$ algebra in the high $\mathrm{T}_{c}$ cuprates [14], operators here have different physical meanings.

These 16 fermion bi-linears are related through the Fierz identity

$$
\sum_{1 \leq a<b \leq 5} L_{a b}^{2}(i)+\sum_{1 \leq a \leq 5} n_{a}^{2}(i)+\frac{5}{4}(n(i)-2)^{2}=5 .
$$

Defining the time reversal operator as $T=R C$, and using the properties of the $R$ matrix given in Eq. (33), it can be shown that $n(i), n_{a}(i)$ are even while $L_{a b}(i)$ is odd under $T$ :

$$
T n T^{-1}=n, \quad T n_{a} T^{-1}=n_{a}, \quad T L_{a b} T^{-1}=-L_{a b} .
$$

On the other hand, we can relate the above $\Gamma$-matrices with the usual spin $S U(2)$ operators $J_{i}$, which form a subgroup of the $S O(5)$ group

$$
\begin{aligned}
J_{ \pm} & =J_{x} \pm i J_{y} \\
& =\sqrt{3}\left(-L_{34} \pm i L_{24}\right)+\left(L_{12} \pm i L_{25}\right) \mp i\left(L_{13} \pm i L_{35}\right), \\
J_{z} & =-L_{23}+2 L_{15}
\end{aligned}
$$

It is easy to check that the $S_{i}$ operators form the $s=3 / 2$ representation of the $S U(2)$ algebra. While the above equation expresses the spin operators in terms of the $\Gamma$ matrices of the $S O(5)$ algebra, the reverse can also be accomplished. The five $\Gamma^{a}$ matrices can actually be expressed in terms of quadratic forms of the spin matrices:

$$
\Gamma^{a}=\xi_{i j}^{a}\left(S_{i} S_{j}+S_{j} S_{i}\right)
$$

where $\xi_{i j}^{a}$ is a rank-2 symmetric traceless tensor given Eq. (29), and discussed more explicitly in Ref. [15]. The ten anti-symmetric tensor $\Gamma^{a b}$ matrices contain both the three linear (rank-1) $S_{i}$ and seven cubic symmetric traceless (rank-3) combination of $S_{i} S_{j} S_{k}$ operators, and they correspond to the second and the fourth rows of 
Eq. (29). Thus, the $n(i)$ operator describes a particlehole pair with total spin zero, the five $n_{a}(i)$ operators describe five particle-hole pair states with total spin two, and the ten $L_{a b}$ operators include the degenerate three spin-1 and seven spin-3 particle-hole pair states. From this point of view, the physical meaning of Eq. (39) and Eq. (29) becomes transparent: operators with even total spins are even under $T$, while operators with odd total spins are odd under $T$.

Using the identities

$$
\begin{aligned}
& \left(\Gamma^{a} R\right)_{\alpha \beta}\left(R \Gamma^{a}\right)_{\gamma \delta}=\frac{5}{4} \delta_{\alpha \gamma} \delta_{\beta \delta}-\frac{3}{4} \Gamma_{\alpha \gamma}^{a} \Gamma_{\beta \delta}^{a}-\frac{1}{4} \Gamma_{\alpha \gamma}^{a b} \Gamma_{\beta \delta}^{a b}, \\
& R_{\alpha \beta} R_{\gamma \delta}=\frac{1}{4} \delta_{\alpha \gamma} \delta_{\beta \delta}+\frac{1}{4} \Gamma_{\alpha \gamma}^{a} \Gamma_{\beta \delta}^{a}-\frac{1}{4} \Gamma_{\alpha \gamma}^{a b} \Gamma_{\beta \delta}^{a b},
\end{aligned}
$$

and the Firez identity Eq. (38), we can now express the $s=3 / 2$ Hubbard model in the following form:

$$
\begin{aligned}
H_{K} & =-t \sum_{i}\left(\psi_{i, \sigma}^{\dagger} \psi_{j, \sigma}+\text { h.c. }\right)-\sum_{i} \mu \psi_{i \sigma}^{\dagger} \psi_{i \sigma} \\
H_{I} & =-\sum_{i, 1 \leq a \leq 5}\left\{\frac{g_{c}}{2}(n(i)-2)^{2}+\frac{g_{v}}{2} n_{a}^{2}(i)\right\}
\end{aligned}
$$

where

$$
\begin{aligned}
& g_{c}=-\left(3 U_{0}+5 U_{2}\right) / 8 \\
& g_{v}=\left(U_{2}-U_{0}\right) / 2
\end{aligned}
$$

\section{B. Absence of the sign problem}

After a series of transformations, we arrived at a form of the $s=3 / 2$ Hubbard which is suitable for the $T$ invariant decomposition method. The interactions in Eq. (43) are fully expressed in $T$-invariant fermion operators in the particle-hole channel. When $g_{c}, g_{v} \geq 0$, i.e,

$$
-3 / 5 U_{0} \geq U_{2} \geq U_{0}
$$

the partition function can be expressed using the $T$ invariant decomposition as

$$
\begin{aligned}
& Z=\int D \psi^{\dagger} D \psi \exp \left\{-\int_{0}^{\beta} d \tau \psi_{\sigma}^{\dagger}\left(\frac{\partial}{\partial \tau}+H\right) \psi_{\sigma}\right\} \\
& =\int D n \int D n^{a} \exp \left\{-\frac{g_{c}}{2} \int_{0}^{\beta} d \tau \sum_{i}(n(i, \tau)-2)^{2}\right. \\
& \left.-\frac{g_{v}}{2} \int_{0}^{\beta} d \tau \sum_{i, a} n_{a}^{2}(i, \tau)\right\} \operatorname{det}\{I+B\}
\end{aligned}
$$

Again $I+B=I+\mathcal{T} e^{-\int_{0}^{\beta} d \tau\left(H_{K}+H_{i}(\tau)\right)}$ is obtained from the integration of fermion fields, $n$ and $n_{a}$ are real HS bose fields. The time-dependent interaction $H_{I}(\tau)$ after the HS transformation is

$$
\begin{aligned}
H_{I}(\tau)= & -g_{c} \sum_{i} \psi_{i, \sigma}(\tau) \psi_{i, \sigma}(\tau) n(i, \tau) \\
& -g_{v} \sum_{i, a} \psi_{i, \sigma}^{\dagger}(\tau) \Gamma_{\sigma, \sigma^{\prime}}^{a} \psi_{i, \sigma^{\prime}}(\tau) n_{a}(i, \tau) .
\end{aligned}
$$

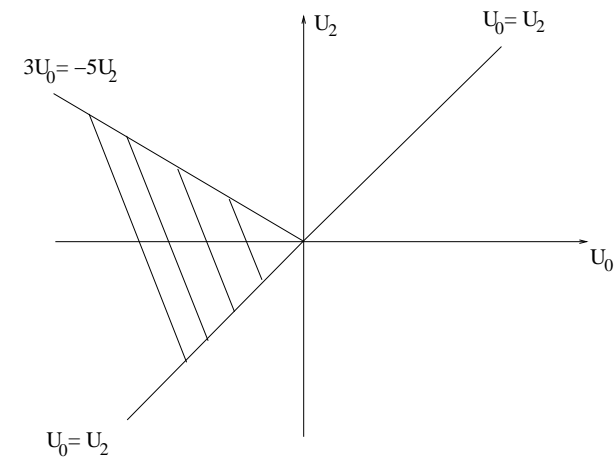

FIG. 2: Within the method of $T$-invariant decomposition, the shaded area marks the parameter region without the sign problem in the $s=3 / 2$ Hubbard model at any doping level and lattice geometry. The fermion determinant can only be factorized along the $S U(4)$ line with $U_{0}=U_{2}<0$, where the traditional algorithms can be applied without the sign problem. The sign problem along the line with $U_{0}=U_{2}>0$ only disappears at the half-filling and on a bipartite lattice.

We see that $H_{I}(\tau)$ mixes the four spin components together, therefore the fermion determinant is factorizable if and only if $g_{v}=0$, which is the $S U(4)$ line with $U_{0}=U_{2}<0$. We define the time reversal transformation $T$ for the entire lattice as $T=\left(\prod_{i} \otimes R(i)\right) C$. From Eq. (39) we see that both terms are $T$-invariant:

$$
T\left(H_{K}+H_{I}\right) T^{-1}=H_{K}+H_{I},
$$

and all other conditions of our theorem are met. Therefore, the minus sign is absent as long as Eq. (45D) is satisfied. This is a much broader parameter range shown in Fig. 2 compared to the conventional factorizibility condition $U_{0}=U_{2}<0$. Our algorithm therefore enables us to study the $s=3 / 2$ Hubbard model away from the $S U(4)$ line. Our proof is valid for any filling level and lattice topology. In this parameter range, it is shown in Ref. [1] that a number of interesting competing orders such as the staggered order of $n^{a}$, singlet superconductivity, and the charge density wave can exist there.

At half-filling and on a bipartite lattice where $\mu=0$, the sign problem also disappears along the $S U(4)$ line at $U=U_{0}=U_{2}>0$. Similar to the spin $1 / 2$ case, after performing a partial particle-hole transformation

$$
c_{i \frac{-1}{2}} \rightarrow(-)^{i} c_{i \frac{-1}{2}}^{\dagger}, \quad c_{i \frac{-3}{2}} \rightarrow(-)^{i} c_{i \frac{-3}{2}}^{\dagger},
$$

while keeping $c_{i \frac{3}{2}}, c_{i \frac{1}{2}}$ operators unchanged. The kinetic energy part is invariant under the above transformation, while the interaction part is change into $H_{\text {int }}=$ $2 U \sum_{i} L_{15}^{2}(i)$. It can be decomposed using the imaginary number as

$$
Z=\int D Q \exp \left\{-2 U \int_{0}^{\beta} d \tau Q^{2}(i)\right\} \operatorname{det}\{I+B\}
$$

where $B=\mathcal{T} e^{-\int_{0}^{\beta} d \tau H_{K}+H_{i}(\tau)}$ with $H_{i}(\tau)=$ $i U \sum_{i} \psi_{i}^{\dagger}(\tau) \Gamma^{15} \psi_{i}(\tau) Q(i, \tau)$. Because $T\left(i L_{15}\right) T^{-1}=$ 
$i L_{15}$, the $\operatorname{det}(I+B)$ is positive definite. However, we did not succeed to generalize this at negative values of $g_{c}$ or $g_{v}$ away from the $S U(4)$ line at half-filling.

In practice, it is more efficient to sample with discrete HS transformation using two Ising-like fields $\eta, s$ for each quartic fermion term as in Ref. [16] instead of using the continuous HS boson field. For any bilinear fermionic operator $O(i)$, the decomposition below has the numerical precision at the order of $O(\Delta \tau)^{4}$ as

$$
\begin{aligned}
& e^{g \Delta \tau \hat{O}(i, \tau)^{2}}=\sum_{l, s= \pm 1} \frac{\gamma_{l}}{4} e^{s \eta_{l} \sqrt{\Delta \tau g} \hat{O}(i, \tau)}+O\left(\Delta \tau^{4}\right), \\
& e^{-g \Delta \tau \hat{O}(i, \tau)^{2}}=\sum_{l, s= \pm 1} \frac{\gamma_{l}}{4} e^{i s \eta_{l} \sqrt{\Delta \tau g} \hat{O}(i, \tau)}+O\left(\Delta \tau^{4}\right),
\end{aligned}
$$

where $g>0$ and $\gamma_{l}=1+\frac{\sqrt{6}}{3} l, \eta_{l}=\sqrt{2(3-\sqrt{6} l)}$. The above proof for the positive definite of $\operatorname{det}(I+B)$ applies equally well in this scheme.

We only used the time reversal properties of the $S O(5)$ algebra in the above proof; the exact $S O(5)$ symmetry is useful for transforming the model expressed in the particle-particle channel to the particle-hole channel, but is not essential. A general anisotropic spin 3/2 lattice model is defined by

$$
\begin{aligned}
H & =-\sum_{\langle i j, a\rangle}\left\{t \psi_{i \alpha}^{\dagger} \psi_{j \alpha}+t_{a} \psi_{i \alpha}^{\dagger} \Gamma_{\alpha \beta}^{a} \psi_{j \beta}+h . c .\right\} \\
& +\sum_{i, a}\left\{h_{a} n_{a}(i)-\mu n(i)\right\}+\sum_{i, a<b}\left\{-\frac{g_{c}}{2}(n(i)-2)^{2}\right. \\
& \left.-\frac{g_{a}}{2} n_{a}^{2}(i)+\frac{g_{a b}}{2} L_{a b}^{2}(i)\right\},
\end{aligned}
$$

where $t_{a}$ is the spin dependent hopping amplitude, $h_{a}$ is the analogy of the Zeeman field coupling to the $n_{a}(i)$ field, $g_{a}$ and $g_{a b}$ are coupling constants in corresponding channels. When $g_{c}, g_{a}, g_{a b}$ are arbitrary positive interaction parameters, we can perform the same decomposition process as before. By using the fact that $T\left(i L_{a b}\right) T^{-1}=i L_{a b}$, we again reach the positive definite fermion determinant. This conclusion also holds for any valid representation of $\Gamma$ matrices, with the redefined $n(i), n_{a}(i), L_{a b}(i)$ and time reversal operations accordingly.

\section{General higher spin Hubbard models}

We can generalize the results in the spin $3 / 2$ case to the any fermionic system with spin $s=n-\frac{1}{2}$. The spin $s=n-\frac{1}{2}$ Hubbard model can be written as

$$
\begin{aligned}
& H=-t \sum_{\langle i j\rangle, \sigma}\left\{c_{i \sigma}^{\dagger} c_{j \sigma}+h . c .\right\}-\left(\mu+\mu_{0}\right) \sum_{i \sigma} c_{i \sigma}^{\dagger} c_{i \sigma} \\
& +\sum_{i, J, J_{z}} U_{J} P_{J J_{z}}^{\dagger}(i) P_{J J_{z}}(i)
\end{aligned}
$$

where $J=0,2, \ldots, 2 n-2$ are the total spin of the particleparticle pairs, $J_{z}=0, \pm 1, \ldots, \pm J$. The pairing operators $P_{J, J_{z}}^{\dagger}$ are defined through the Clebsch-Gordan coefficient for two indistinguishable particles as

$$
P_{J, J_{z}}^{\dagger}(i)=\sum_{\alpha \beta}\left\langle J, J_{z} \mid s, s ; \alpha \beta\right\rangle c_{\alpha}^{\dagger}(i) c_{\beta}^{\dagger}(i)
$$

The total spin of the particle-particle pair takes only even integer values so that Pauli principle is satisfied on every site. At half-filling and on a bipartite lattice, $\mu=0$ ensures the particle-hole symmetry, and $\mu_{0}=1 /(2 n) \sum_{J}(2 J+1) U_{J}$.

The general strategy to implement the method of $T$ invariant decomposition is to first transform the interaction terms originally expressed in the particle-particle channel to the particle-hole channel. In this case, we have fermion operators $\psi_{i, \beta}$ within one unit cell, where $\beta=1, . .,(2 s+1)$. Therefore, there are $(2 s+1)^{2}$ fermion bi-linears, of the form $M^{I}=\psi_{i, \alpha}^{\dagger} M_{\alpha \beta}^{I} \psi_{i, \beta}$, where $I=$ $1, \ldots,(2 s+1)^{2}$. The $(2 s+1)^{2} M_{\alpha \beta}^{I}$ matrices can in general be expressed in a complete basis in terms of the product of spin $s$ matrices $S_{i}$ :

$$
\begin{aligned}
& 1, \\
& S^{i}, \quad i=1,2,3, \\
& \xi_{i j}^{a} S_{i} S_{j}, \quad a=1, . ., 5, \\
& \ldots \\
& \xi_{i_{1}, i_{2}, \ldots i_{J}}^{L} S_{i_{1}} S_{i_{2}} \ldots S_{i_{J}}, \quad L=1, . .,(4 s+1),
\end{aligned}
$$

where $\xi$ 's are fully symmetric, traceless tensors, satisfying

$$
\xi_{i_{1}, i_{2}, \ldots i_{J}}^{L}=\xi_{i_{2}, i_{1}, \ldots i_{J}}^{L}
$$

or any other permutation of indices, and

$$
\xi_{i_{1}, i_{1}, \ldots i_{J}}^{L}=0
$$

Spherical harmonics can be used to explicitly construct these tensors [17]. This decomposition is obviously complete, since

$$
(2 s+1)^{2}=1+3+5+\ldots+(4 s+1)
$$

According to the method of $T$-invariant decomposition, any negative interaction terms in the even spin channel like $1, \xi_{i j}^{a} S_{i} S_{j}, \ldots$ or any positive interaction terms in the odd spin channel like $S_{i}, \xi_{i j k}^{L} S_{i} S_{j} S_{k}, \ldots$ can be simulated by our algorithm without the sign problem.

In the following, we shall illustrate this general procedure more explicitly for a special case of the higher spin Hubbard model where

$$
U_{2}=U_{4}=\ldots=U_{2 n-2} \equiv U^{\prime} .
$$

The generic higher spin Hubbard model only has the spin $S U(2)$ symmetry for $s \neq \frac{3}{2}$. However, under the above 
condition, the higher spin Hubbard has the $S p(2 n)$ symmetry. When an additional condition, namely $U_{0}=U^{\prime}$ is imposed, the model has a larger, $S U(2 n)$ symmetry. In Appendix $\mathrm{A}$ an introduction to the $S p(2 n)$ algebra is given. As shown there, the singlet pairing operator is also the singlet of the $S p(2 n)$ group, while all other $2 n^{2}-n-1$ pairing operators with $J=2,4, \ldots, 2 n-2$ together form a representation for the $S p(2 n)$ group. Thus we conclude that Eq. (51) is $S p(2 n)$ symmetric if and only if coupling constants satisfy Eq. (57). For $\mathrm{n}=1$ and 2, the $S p(2 n)$ symmetry is generic and does not need any fine-tuning. Actually, $S p(2)$ is isomorphic to $S U(2)$, while $S p(4)$ is isomorphic to $S O(5)$. This is consistent with our earlier finding that the $s=3 / 2$ Hubbard model has the $S O(5)$, or the $S p(4)$ symmetry without any conditions on the parameters [1].

To show the $S p(2 n)$ symmetry explicitly, we can rewrite the Hamiltonian in Eq, (51) as

$$
\begin{aligned}
H & =-t \sum_{\langle i j\rangle, \sigma}\left\{\psi_{i, \alpha}^{\dagger} \psi_{j, \alpha}+\text { h.c. }\right\}-\mu \sum_{i} \psi_{i, \alpha}^{\dagger} \psi_{i, \alpha} \\
& +c_{0} \sum_{i}\left(\psi_{i, \alpha}^{\dagger} \psi_{i, \alpha}-n\right)^{2}+c_{2} \sum_{i}\left(\psi_{i, \alpha}^{\dagger} Y_{\alpha \beta}^{a} \psi_{i, \beta}\right)^{2}, \\
c_{0} & =\frac{n+1}{4 n^{2}} U_{0}+\frac{2 n^{2}-n-1}{4 n^{2}} U^{\prime}, \quad c_{2}=\frac{U_{0}-U^{\prime}}{2 n} .(58)
\end{aligned}
$$

The expression for $Y^{a}\left(1 \leq a \leq 2 n^{2}-n-1\right)$ is given in Ap- pendix where it is also shown that they are even under the time-reversal transformation. By the same reasoning before, we perform the HS decoupling in the above two channels. Then the sign problem is absent at both $c_{1}$ and $c_{2}$ are negative, i.e.

$$
U_{0} \leq U^{\prime} \leq-\frac{n+1}{2 n^{2}-n-1} U_{0}
$$

Because the $Y^{a}\left(1 \leq a \leq 2 n^{2}-n-1\right)$ are even under timereversal transformation while the spin operators $S_{i}(i=$ $x, y, z)$ are odd, $Y^{a}$ can be expanded in the basis of Eq. (53) including all terms with even powers of spin matrix.

\section{BI-LAYER $\mathrm{S}=1 / 2$ MODELS}

The spin 3/2 Hubbard model has a close relationship with a bi-layer model introduced by Scalapino, Zhang and Hanke(SZH) 11]. This model was constructed and extensively investigated because of the exact particleparticle channel $S O(5)$ symmetry between the antiferromagnetism and superconductivity when the coupling constants satisfy a simple relation $18,19,20,21]$. The original SZH model was introduced on a two leg ladder, and it is straightforward to generalize it to a bi-layer system as

$$
\begin{aligned}
H & =-t_{\|} \sum_{\langle i j\rangle}\left\{c_{i \sigma}^{\dagger} c_{j \sigma}+d_{i \sigma}^{\dagger} d_{j, \sigma}+\text { h.c. }\right\}-t_{\perp} \sum_{i}\left\{c_{i, \sigma}^{\dagger} d_{i, \sigma}+\text { h.c. }\right\}-\mu \sum_{i}\left\{n_{c}(i)+n_{d}(i)\right\} \\
& +U \sum_{i}\left\{\left(n_{\uparrow, c}(i)-\frac{1}{2}\right)\left(n_{\downarrow, c}(i)-\frac{1}{2}\right)+\left(n_{\uparrow, d}(i)-\frac{1}{2}\right)\left(n_{\downarrow, d}(i)-\frac{1}{2}\right)\right\} \\
& +V \sum_{i}\left(n_{c}(i)-1\right)\left(n_{d}(i)-1\right)+J \sum_{i} \vec{S}_{i, c} \cdot \vec{S}_{i, d},
\end{aligned}
$$

where $c^{\dagger}$ and $d^{\dagger}$ are creation operators in the upper and lower layer respectively, $t_{\|}$and $t_{\perp}$ are the hopping amplitude in the layer and cross the rung respectively, $U$ is the onsite interaction, $V$ and $J$ are the charge and Heisenberg exchange interaction across the rung respectively. The SZH model is known to have an exact $S O(5)$ symmetry when

$$
J=4(U+V), \quad \mu=0,
$$

which unifies antiferromagnetism with superconductivity 11]. Remarkably, there exists another exact $S O(5)$ symmetry in the particle-hole channel when

$$
J=4(U-V), \quad t_{\perp}=0,
$$

and the symmetry is valid for all filling factors. We denote the former particle-particle $S O(5)$ symmetry as

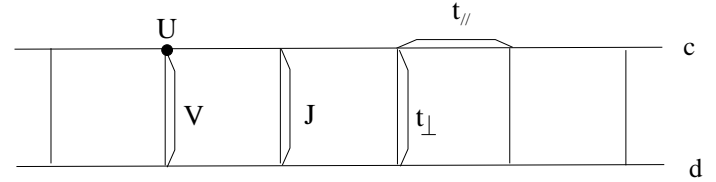

FIG. 3: The SZH model defined on a two-leg ladder segment of the double-layer spin $1 / 2$ system.

$S O(5)_{p p}$ and the later p-h $S O(5)$ symmetry as $S O(5)_{p h}$. The two $S O(5)$ symmetric lines are shown in Fig. 4 In order to employ the method of $T$-invariant decomposition, we adopt the view from the $S O(5)$ symmetry in the particle-hole channel in this section.

There are four single fermion states per unit cell in both the $s=3 / 2$ Hubbard model and the $s=$ 


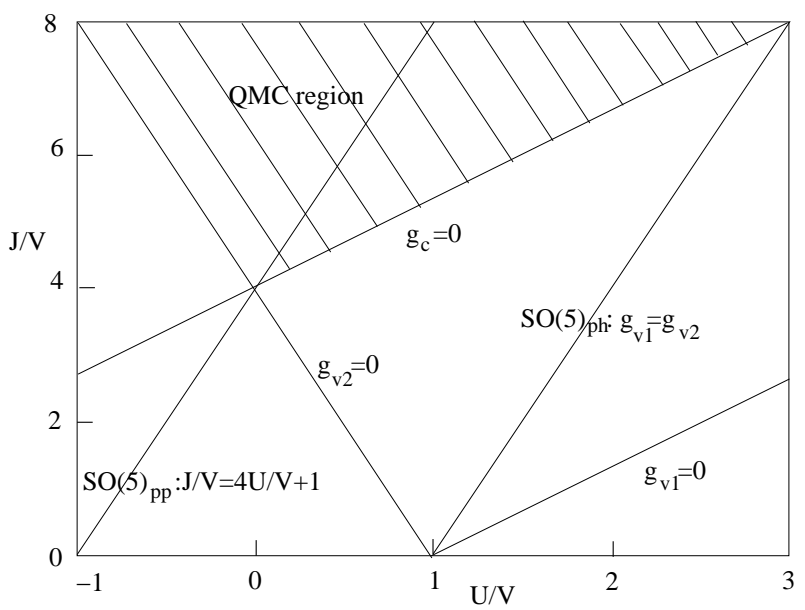

FIG. 4: Two $S O(5)$ lines are shown in the SZH model as well as the QMC region without the sign problem for any filling (hatched area): $g_{v 1}>0, g_{v 2}>0$ and $g_{c}>0$. There is another region with $V<0$ (not shown).

1/2 bi-layer model, a mapping between them can be established through $\psi_{i}=\left(c_{i, \frac{3}{2}}, c_{i, \frac{1}{2}}, c_{i,-\frac{1}{2}}, c_{i,-\frac{3}{2}}\right)^{T} \leftrightarrow$ $\left(c_{i, \uparrow}, c_{i, \downarrow}, d_{i, \uparrow}, d_{i, \downarrow}\right)^{T}$. We denote the time reversal operator defined in Eq. (34) for the $s=\frac{3}{2}$ system as $T_{1}$, and the usual definition for $s=1 / 2$ system as $T_{2}$. $T_{1}$ actually is the combined operation of $T_{2}$ and the interchange between the upper and lower layers:

$$
T_{1}=\left(\begin{array}{ll}
0 & I \\
I & 0
\end{array}\right) T_{2}
$$

The $16 \mathrm{p}$-h channel fermionic bilinear forms are mapped onto

$$
\begin{aligned}
n(i) & =c_{i \sigma}^{\dagger} c_{i \sigma}+d_{i \sigma}^{\dagger} d_{i \sigma} \\
n_{1}(i) & =-i\left(d_{i \sigma}^{\dagger} c_{i \sigma}-h . c .\right) / 2 \\
n_{5}(i) & =\left(d_{i \sigma}^{\dagger} c_{i \sigma}+h . c\right) / 2 \\
n_{2,3,4}(i) & =c_{i \alpha}^{\dagger}\left(\frac{\vec{\sigma}}{2}\right)_{\alpha \beta} c_{i \beta}-d_{i \alpha}^{\dagger}\left(\frac{\vec{\sigma}}{2}\right)_{\alpha \beta} d_{i \beta} \\
\operatorname{Re} \vec{\pi}(\mathrm{i}) & =c_{i \alpha}^{\dagger}\left(\frac{\vec{\sigma}}{2}\right)_{\alpha \beta} d_{i \beta}+h . c . \\
\operatorname{Im} \vec{\pi}(\mathrm{i}) & =-i\left(c_{i \alpha}^{\dagger}\left(\frac{\vec{\sigma}}{2}\right)_{\alpha \beta} d_{i \beta}-h . c .\right) \\
\vec{S}_{i} & =c_{i \alpha}^{\dagger}\left(\frac{\vec{\sigma}}{2}\right)_{\alpha \beta} c_{i \beta}+d_{i \alpha}^{\dagger}\left(\frac{\vec{\sigma}}{2}\right)_{\alpha \beta} d_{i \beta} \\
Q & =\left(n_{c}(i)-n_{d}(i)\right) / 2,
\end{aligned}
$$

where $n_{1,5}$ are the singlet rung current and rung bond order parameters respectively, and $\operatorname{Im} \vec{\pi}$ and $\operatorname{Re} \vec{\pi}$ are their triplet counterpart; $n_{2,3,4}$ are the rung Neel order parameter, and $\vec{S}$ is the total rung spin, $Q$ is charge density wave order parameter. $n, n_{1 \sim 5}$ are even under the $T_{1}$ transformation and the others are odd. In contrast, $n$, $n_{5}, \operatorname{Im} \vec{\pi}$ and $Q$ are even under the usual definition $T_{2}$ and the others are odd.
The general SZH model can be mapped into an anisotropic $S O(5)$ model in the following form:

$$
\begin{aligned}
H & =-t_{\|} \sum_{\langle i j\rangle} \psi_{i \alpha}^{\dagger} \psi_{j \alpha}+t_{\perp} \sum_{i} \psi_{i \alpha}^{\dagger} \Gamma_{\alpha \beta}^{5} \psi_{i \beta}-\mu \sum_{i} n_{i}, \\
& +\sum_{i}\left\{-\frac{g_{c}}{2}(n(i)-2)^{2}-\frac{g_{v 1}}{2}\left(n_{1}^{2}(i)+n_{5}^{2}(i)\right)-\frac{g_{v 2}}{2}\right. \\
& \times\left(n_{2}^{2}(i)+n_{3}^{2}(i)+n_{4}^{2}(i)\right),
\end{aligned}
$$

with

$$
\begin{aligned}
4 g_{c} & =\frac{3}{4} J-U-3 V, \quad 4 g_{v 1}=\frac{3}{4} J-U+V, \\
4 g_{v 2} & =\frac{J}{4}+U-V .
\end{aligned}
$$

The particle-hole channel $S O(5)_{p h}$ symmetry is restored at $g_{v 1}=g_{v 2}$ and $t_{\perp}=0$, i.e., when the conditions of Eq. (62) are satisfied. At this point, the SZH model expressed in Eq. (65) takes exactly the same form as the $s=3 / 2$ Hubbard model expressed in Eq. (43). The equivalence between the two models is therefore rigorously established.

For the general SZH model, the interactions can be expressed purely in terms of the fermion bi-linears which are invariant under $T_{1}$ transformation from Eq. (65), by virtue of Eq. (39). We perform the $T_{1}$ invariant decomposition of the interactions in the region of $g_{c}, g_{v 1}, g_{v 2} \geq 0$, i.e.

$$
\left\{\begin{array}{l}
\frac{3}{4} J+V \geq U \geq-\frac{1}{4} J+V \\
\frac{3}{4} J \geq U+3 V
\end{array}\right.
$$

as shown in Fig. 4 then the sign problem is absent. In this region with positive $g_{v 1,2}$ and $g_{c}$, we expect the competing orders of the five-vector channel and the superconductivity, i.e. the antiferromagnetism, staggered current and the rung-singlet superconductivity, which can be investigated systematically with high numerical accuracy, at and away from the half-filling.

The above algorithm has been applied to demonstrate the existence of 2-dimensional staggered current phase conclusively at half-filling with $t_{\|}=1, t_{\perp}=0.1, U=$ $0, V=0.5, J=2[10]$. The current pattern is illustrated in Fig. [5 with staggered inter-layer currents (SIC) between the bi-layers and alternating source to drain currents within the bi-layers. Viewed from the top, this current pattern has a $s$-wave symmetry. While the Ddensity wave 22] currents are divergence free within the layer, the SIC current is curl free within the layer. These two patterns can be considered as dual to each other in two dimensions. As far as in our knowledge, this is the first time a current carrying ground state has been conclusively demonstrated in a $2 \mathrm{D}$ system.

The mapping between the SZH model and the $s=3 / 2$ model are not unique. More generally the SZH model can be written as

$$
H=-t_{\|} \sum_{\langle i j\rangle} \psi_{i \alpha}^{\dagger} \psi_{j \alpha}+t_{\perp} \sum_{i} \psi_{i \alpha}^{\dagger} \Gamma_{\alpha \beta}^{5} \psi_{i \beta}-\mu \sum_{i} n_{i},
$$


(b)

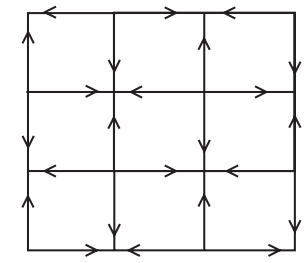

(a)
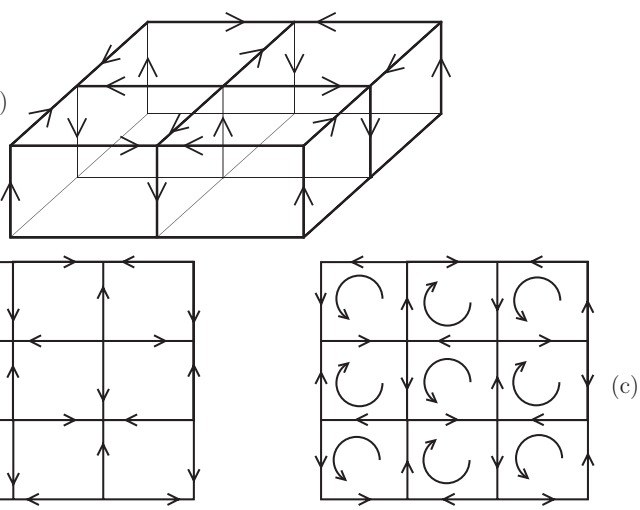

FIG. 5: (a) Sketch of a staggered interlayer current phase from Ref. [10]. For clarity, we do not show the bottom layer current. (b)Top view of the bi-layer current. (c) Sketch of the D-density wave current pattern for comparison.

$$
\begin{aligned}
& +\sum_{i}\left\{-\frac{g_{c}}{2}(n(i)-2)^{2}-\frac{g_{v 1}}{2}\left(n_{1}^{2}(i)+n_{5}^{2}(i)\right)-\frac{g_{v 2}}{2}\right. \\
& \times\left(n_{2}^{2}(i)+n_{3}^{2}(i)+n_{4}^{2}(i)\right)+\frac{g_{t 1}}{2} Q^{2}(i)+\frac{g_{t 2}}{2}(\vec{S}(i) \cdot \vec{S}(i)) \\
& +\frac{g_{t 3}}{2}(\operatorname{Re} \vec{\pi}(\mathrm{i}) \cdot \operatorname{Re} \vec{\pi}(\mathrm{i})+\operatorname{Im} \vec{\pi}(\mathrm{i}) \cdot \operatorname{Im} \vec{\pi}(\mathrm{i})) .
\end{aligned}
$$

Only three out of the six coupling constants are independent, as shown here in the correspondence to the $U, V, J$ parameters

$$
\begin{aligned}
U & =-4 g_{c}+3 g_{v 2}+g_{t 1}-3 g_{t 2} \\
V & =-4 g_{c}+g_{v 1}-g_{t 1}-3 g_{t 3} \\
J & =4\left(g_{v 1}+g_{v 2}+g_{t 2}+g_{t 3}\right) .
\end{aligned}
$$

If the $g_{t 1}, g_{t 2}, g_{t 3}$ are set to zero, it returns to Eq. (65). For any given values for $U, V, J$, if we can find a set of value of $g_{c}, g_{v 1}, g_{v 2}, g_{t 1}, g_{t 2}, g_{t 3} \geq 0$, then we can perform the HS transformation keeping the invariance under the $T_{1}$ operation and arrive at the absence of the sign problem regardless the doping and lattice topology. This general decoupling scheme extends the valid parameter region in Eq. (67). On the other hand, we can also consider to perform HS decoupling with the invariance under the usual definition of the time reversal operation $T_{2}$. After setting $g_{v 1}, g_{t 3}=0$, we have the condition that $g_{c} \geq 0, g_{v 2} \leq 0, g_{t 1} \leq 0, g_{t 2} \geq 0$. This decoupling scheme based $T_{1}$ also enlarges the region of Eq. (67). For example, the usual bilayer negative $U$ Hubbard model with $U<0, V=J=0$ is out of that region. Nevertheless, we can still show the absence of the sign problem by setting $g_{c}=-g_{t 1} / 4=-U / 2>0$ and all other parameters zero.

In contrast, the conventional algorithm based on the factorization of the fermion determinant works only at either $g_{v 1}=g_{v 2}=0, g_{c}<0$ or the usual negative $\mathrm{U}$ Hubbard model $U<0, V=J=0$. This parameter set is included in the above HS decomposition schemes respecting either the $T_{2}$ or the $T_{1}$ time reversal symmetry. We therefore see the significant improvement provided by the method of the $T$-invariant decomposition.

\section{MODELS WITH BOND INTERACTIONS}

So far, the models we considered only have on-site interactions. In this section, we will generalize them to include interactions defined on the bond. Such models can have many exotic phases.

We first consider the following general single layer spin 1/2 Hamiltonian with bond interactions

$$
\begin{aligned}
H & =-t \sum_{\langle i j\rangle, \sigma}\left(c_{i \sigma}^{\dagger} c_{j \sigma}+h . c .\right)-\mu \sum_{i} n(i) \\
& +\sum_{\langle i j\rangle}\left\{-\frac{g_{s b d}}{2} M_{i j} M_{i j}+\frac{g_{s c u r}}{2} N_{i j} N_{i j}\right. \\
& \left.+\frac{g_{t b d}}{2} \vec{M}_{i j} \cdot \vec{M}_{i j}-\frac{g_{t c u r}}{2} \vec{N}_{i j} \cdot \vec{N}_{i j}\right\}, \\
\vec{M}_{i j} & =c_{i \alpha}^{\dagger}\left(\frac{\vec{\sigma}}{2}\right)_{\alpha \beta} c_{j \beta}+h . c ., \quad \vec{N}_{i j}=i\left\{c_{i \alpha}^{\dagger}\left(\frac{\vec{\sigma}}{2}\right)_{\alpha \beta} c_{j \beta}-\text { h.c. }\right\}, \\
M_{i j} & =c_{i \sigma}^{\dagger} c_{j \sigma}+h . c ., \quad N_{i j}=i\left\{c_{i \sigma}^{\dagger} c_{j \sigma}-\text { h.c. }\right\},
\end{aligned}
$$

where $M_{i j}$ and $N_{i j}$ are the singlet bond and current operators on the bond $\langle i j\rangle, \vec{M}_{i j}$ and $\vec{N}_{i j}$ are their triplet counterparts, $g_{s b d}, g_{s c u r}, g_{t b d}$ and $g_{t c u r}$ are the coupling constants in the corresponding channels. The sites $i$ and $j$ forming the bond $\langle i j\rangle$ are not necessary nearest neighbors, but can be at arbitrary distance apart. Under the time reversal transformation $T, M_{i j}$ and $\vec{N}_{i j}$ are even while $\vec{M}_{i j}$ and $N_{i j}$ are odd.

These four interactions are not independent, and can be reorganized into

$$
\begin{aligned}
H_{\text {int }} & =\sum_{\langle i j\rangle}\left\{-J_{c}\left(c_{i \uparrow}^{\dagger} c_{i \downarrow}^{\dagger} c_{j \downarrow} c_{j \uparrow}+\text { h.c. }\right)\right. \\
& \left.+V(n(i)-1)(n(j)-1)+J_{s} \vec{S}(i) \cdot \vec{S}(j),\right\} \\
J_{c} & =2\left(g_{s b d}+g_{\text {scur }}\right)+3\left(g_{t b d}+g_{t c u r}\right), \\
V & =\frac{g_{\text {sbd }}-g_{\text {scur }}}{2}-\frac{3}{4}\left(g_{t b d}-g_{t c u r}\right), \\
J_{s} & =2\left(g_{\text {sbd }}-g_{\text {scur }}\right)+\left(g_{t b d}-g_{\text {tcur }}\right) .
\end{aligned}
$$

The $J_{c}$ term is the pair hopping, $V$ is the charge interaction between site $i$ and $j$, and $J_{s}$ is the Heisenberg exchange. When all of $g_{s b d}, g_{s c u r}, g_{t b d}, g_{t c u r}$ are positive, we perform the HS decomposition in each channel respectively as 


$$
\begin{aligned}
Z & =\int D M D \vec{M} D N D \vec{N} \exp \left\{-\int_{0}^{\beta} d \tau \sum_{\langle i j\rangle} g_{s b d} M_{i j}^{2}(\tau)+g_{s c u r} N_{i j}^{2}(\tau)+g_{t b d} \vec{M}_{i j}^{2}(\tau)+g_{t c u r} \vec{N}_{i j}^{2}(\tau)^{2}\right\} \\
& \times \operatorname{det}\{I+B\}
\end{aligned}
$$

where $I+B=I+\mathcal{T} e^{-\int_{0}^{\beta} d \tau H_{K}+H_{I}(\tau)}$. $H_{I}(\tau)$ after the HS decoupling is given by

$$
\begin{aligned}
H_{I}(\tau) & =-\sum_{\langle i j\rangle} g_{s b d} M_{i j}(\tau)\left(c_{i, \sigma}^{\dagger} c_{j, \sigma}+\text { h.c. }\right)+i \sum_{\langle i j\rangle} g_{s c u r} N_{i j}(\tau) i\left(c_{i, \sigma}^{\dagger} c_{j, \sigma}-\text { h.c. }\right) \\
& -i \sum_{\langle i j\rangle} g_{t b d} \vec{M}_{i j}(\tau)\left(c_{i \alpha}^{\dagger}\left(\frac{\vec{\sigma}}{2}\right)_{\alpha \beta} c_{j \beta}+\text { h.c. }\right)+\sum_{\langle i j\rangle} g_{t c u r} \vec{N}_{i j}(\tau) i\left(c_{i, \alpha}^{\dagger}\left(\frac{\vec{\sigma}}{2}\right)_{\alpha \beta} c_{j, \beta}-\text { h.c. }\right)
\end{aligned}
$$

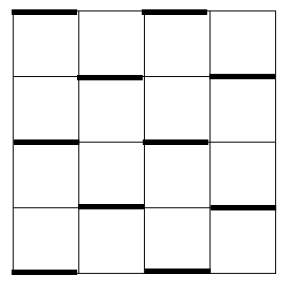

A

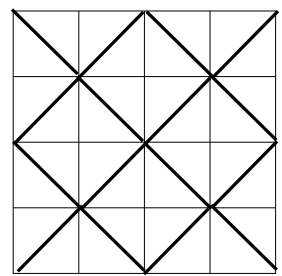

$\mathrm{C}$

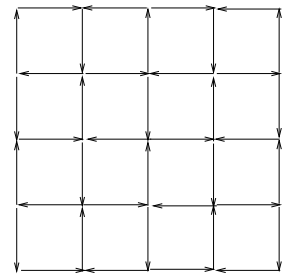

B

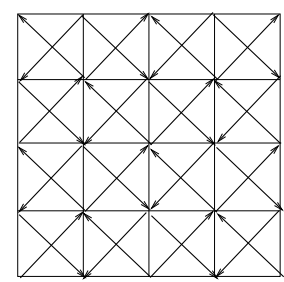

$\mathrm{D}$
FIG. 6: Four possible density-wave phases can be simulated without sign problem. A) singlet spin-Peierls (p-density wave), B) Triplet $d_{x^{2}-y^{2}}$ density wave, C) singlet $d_{x y}$ densitywave, D) Triplet diagonal current.

Therefore, $H_{I}$ and $I+B$ are even under the time-reversal transformation and the sign problem is absent.

The valid parameter region for the above algorithm is very general as long as all $g_{s b d}, g_{s c u r}, g_{t b d}, g_{t c u r} \geq 0$. As a result, $V$ and $J$ can be either positive or negative while $J_{c}$ has to be positive. Many interesting competing orders are supported in this parameter region. For example, various density-wave states exist on a square lattice near half-filling 23] as shown in Fig. [6] With $g_{s b d}, g_{t c u r}>0$, the above algorithm provide a good opportunity to study the singlet bond and the triplet current order parameters formed by $M_{i j}$ and $\vec{N}_{i j}$, while its not good for study the singlet current and the triplet bond order parameters formed by $N_{i j}$ and $\vec{M}_{i j}$ because $g_{t b d}, g_{s c u r}>0$. After setting $g_{t b d}=g_{s c u r}=0$, for the bond $\langle i j\rangle$ connecting the nearest sites, the $g_{s b d}$ term favors the $p$-density wave (spin-Peierls) phase, and the $g_{t c u r}$ term favors the triplet channel $d_{x^{2}-y^{2}}$-density wave. The latter order is recently proposed as the origin as the pseudogap in the high $T_{c}$ cuprates 24]. For the bond interaction between the next nearest bond, i.e. the diagonal bond, the $g_{s b d}$ term leads to the singlet $d_{x y}$ order, and $g_{t c u r}$ term leads to the triplet diagonal current order. The triplet diagonal current phase was studied in the two-leg ladder system using the bosonization method in Ref. [25] and also under the name of the triplet $F$-density wave in Ref. 26].

When the Fermi surface nesting effect is not important either at large doping or in the non-bipartite lattice, the $g_{t c u r}$ term can lead to the $F_{1}^{a}$ channel of the Landau-Pomeranchuk instability on the Fermi surface, which was studied recently in the continuum model in Ref. 27]. After the symmetry breaking, two possible phases are named as $\alpha$ and $\beta$ phases in analogy to the $A$ and $B$ phases in the triplet p-wave channel superfluid phase in ${ }^{3} \mathrm{He}$ as shown in Fig. 7 The $\alpha$-phase was studied by Hirsch [28, 29] under the name of spin-split phase on the lattice system with an opposite anisotropic Fermi surface distortions for two spin components. In contrast, the fermi surface distortion is isotropic and a spin-orbit coupling is dynamically generated in the $\beta$ phase. The two single particle bands are characterized by the helicities. It would be interesting to study these exotic phases in our version of the QMC algorithm free of the sign problems.

Bond interactions can also be added into the spin $3 / 2$ Hubbard model of Eq. (30) as

$$
\begin{aligned}
H_{\text {bond }} & =\sum_{\langle i j\rangle}\left\{-\frac{g_{s b d}}{2} M_{i j} M_{i j}+\frac{g_{s c u r}}{2} N_{i j} N_{i j}\right. \\
& +\sum_{a}-\frac{g_{v b d}}{2} M_{i j}^{a} M_{i j}^{a}+\frac{g_{v s u r}}{2} N_{i j} N_{i j} \\
& \left.+\sum_{a<b} \frac{g_{t b d}}{2} M_{i j}^{a b} M_{i j}^{a b}-\frac{g_{t c u r}}{2} N_{i j}^{a b} N_{i j}^{a b}\right\}, \quad(74) \\
M_{i j} & =\psi_{i}^{\dagger} \psi_{j}+h . c ., \quad N_{i j}=i\left\{\psi_{i}^{\dagger} \psi_{j}-h . c .\right\}, \\
M_{i j}^{a} & =\psi_{i}^{\dagger} \frac{\Gamma^{a}}{2} \psi_{j}+\text { h.c. }, \quad N_{i j}^{a}=i\left\{\psi_{i}^{\dagger} \frac{\Gamma^{a}}{2} \psi_{j}-\text { h.c. }\right\}, \\
M_{i j}^{a b} & =\psi_{i}^{\dagger} \frac{\Gamma^{a b}}{2} \psi_{j}+\text { h.c. }, \quad N_{i j}^{a b}=i\left\{\psi_{i}^{\dagger} \frac{\Gamma^{a b}}{2} \psi_{j}-h . c .\right\},
\end{aligned}
$$




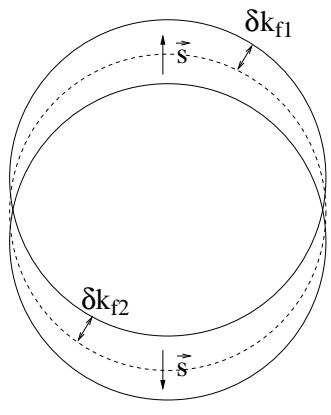

$\alpha-$ phase

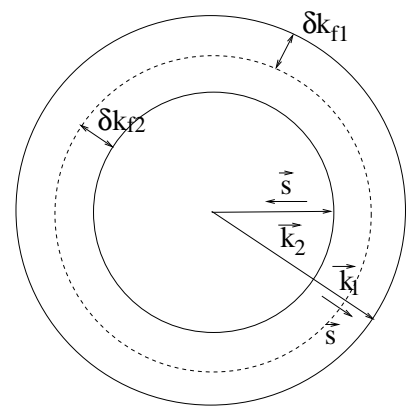

$\beta$-phase
FIG. 7: The fermi surface instability in the $F_{1}^{a}$ channel, with dashed lines marking the fermi surface before symmetry breaking. In the $\alpha$-phase, the anisotropic fermi surface distortion appears for two spin components. In the $\beta$-phase, spin-orbital coupling is generated dynamically and two fermi surfaces are characterized by helicity.

where $M_{i j}$ and $N_{i j}$ are the singlet bond and current operators on the bond $\langle i j\rangle, M_{i j}^{a}, N_{i j}^{a}, M_{i j}^{a b}, N_{i j}^{a b}$ are their 5 -vector and 10-tensor channel counterparts respectively, and $g_{s b d}, g_{v b d}, g_{t b d}, g_{s c u r}, g_{v c u r}, g_{t c u r}$ are the coupling constants in the corresponding channels. Again the site $i$ and $j$ forming the bond $\langle i j\rangle$ can be at an arbitrary distance apart. The bond interactions can be decoupled by introducing the HS field in each channel respectively. Following the same reasoning as the case of the spin $1 / 2$, the bond-interactions keep the fermion determinant positive definite provided all these coupling constant non-negative. Similarly, with $g_{s b d}, g_{v b d}, g_{t c u r}>0$, the algorithm can be applied to study the singlet, quintet bond orders and the 10-fold current order, while with $g_{s c u r}, g_{v c u r}, g_{t b d}>0$, it is not useful for study applied to study the singlet, quintet current orders and the 10-fold bond order.

\section{CONCLUSION}

The sign problem of the fermionic QMC algorithm is one of the most important problems in theoretical physics. Its solution would practically give an universal computational method to solve models with strong correlations. The rigorous theorem established in this work shows that the minus sign problem can be eliminated for a much wider class of models than before, in which the fermion matrix is invariant under an anti-unitary symmetry similar to the time reversal symmetry in quantum mechanics. The method of $T$-invariant decomposition does not only provide a deep connection between the sign problem and the time reversal symmetry, it also leads to practical algorithms which can be applied to many interesting models with strong correlations. Using this algorithm, a new class of models with strong correlation can be simulated, and some novel and exotic ground states have been firmly established.

We conclude this paper with an optimistic outlook. Even though our method can only be applied presently to models with definite constraints among the interaction parameters, we believe that the deep symmetry connections revealed in this work could guide us in future works, and might eventually lead to the complete elimination of the sign problem.

Note added in proof:

After the paper has been accepted, we learned that a similar version of the theorem of T-invariant decomposition had been discussed in the context of lattice gauge theory [30]. However, they did not consider the case that $\mathrm{I}+\mathrm{B}$ is not diagonalizable. Our proof is valid regardless of whether $\mathrm{I}+\mathrm{B}$ is diagonalizable or not, thus is more complete."

\section{Acknowledgments}

We thank Drs. B. A. Bernevig, S. Capponi, D. Ceperley, S. Chandrasekharan, J. P. Hu, D. Scalapino, and T. Xiang for helpful discussions. This work is supported by the NSF under grant numbers DMR-0342832 and the US Department of Energy, Office of Basic Energy Sciences under contract DE-AC03-76SF00515. CW is also supported by the Stanford Graduate Fellowship program.

\section{APPENDIX A: $S p(2 n)$ ALGEBRA IN THE SPIN $s=n-1 / 2$ FERMION SYSTEM}

We give a brief introduction to the $S p(2 n)$ algebra here. The $2 n$-dimensional Hilbert space on each site can be arranged as a direct product between a $n$-dimensional and a 2-dimensional space. The complete basis of eigenstates of $S_{z}$ are labeled in the sequence of $|1\rangle=\left|n-\frac{1}{2}\right\rangle,|2\rangle=$ $\left|-n+\frac{3}{2}\right\rangle, \ldots,|n\rangle=\left|\frac{(-)^{n-1}}{2}\right\rangle$, and $|\overline{1}\rangle=\left|-n+\frac{1}{2}\right\rangle,|\overline{2}\rangle=$ $\left|n-\frac{3}{2}\right\rangle, \ldots,|\bar{n}\rangle=\left|\frac{(-)^{n}}{2}\right\rangle$. The $S p(2 n)$ spinor is defined as

$$
\psi=\left(c_{n-\frac{1}{2}}, c_{-n+\frac{1}{2}}, c_{-n+\frac{3}{2}}, c_{n-\frac{3}{2}}, \ldots\right)^{T} .
$$

Group elements of $S p(2 n)$ include any $2 n \times 2 n$ unitary matrix $U$ satisfying $U^{T} R U=R$ or equivalently $\left.R^{-1} U R=U^{*} 31\right]$ with the $R$-matrix

$$
R=I_{n} \otimes\left(-i \sigma_{2}\right) .
$$

The $R$ matrix is a straightforward generalization of the $R=-i \sigma_{2}$ in the spin $1 / 2$ case, which also satisfies $R^{T}=R^{-1}=R^{\dagger}=-R$. Clearly, the $S p(2 n)$ group is a subgroup of the $S U(2 n)$ group defined in the $2 n$ dimensional space.

In the particle-hole channel, there are $4 n^{2}$ independent bilinear operators as $\psi_{\alpha}^{\dagger} \psi_{\beta}(\alpha=1, \ldots, 2 n, \beta=1, \ldots, 2 n)$. Among them, the particle density operator $n=\psi_{\alpha}^{\dagger} \psi_{\alpha}$ is 
a singlet under both the $S U(2 n)$ and the $S p(2 n)$ group. The time reversal transformation $T=C R$ is defined as usual, and it satisfies $T^{2}=-1$. The other $4 n^{2}-1$ bilinear operators form the generators (adjoint representation) for the $S U(2 n)$ group. They can be decomposed into two classes according to their transformation properties under the $T$ operation. The first class contains $n(2 n+1)$ elements which forms the generators of the $S p(2 n)$ group as denoted as $\psi_{\alpha}^{\dagger} X_{\alpha \beta}^{b} \psi_{\beta}\left(b=1 \sim 2 n^{2}+n\right)$. $X^{b}$ can be expressed in terms a direct product between the $S U(n)$ and $S U(2)$ generators. We define the $S U(n)$ generators as

$$
\begin{aligned}
& \left(M_{i j}^{(1)}\right)_{l k}=\frac{1}{2}\left(\delta_{i l} \delta_{j k}+\delta_{i k} \delta_{j l}\right) \quad(1 \leq i<j \leq n), \\
& \left(M_{i j}^{(2)}\right)_{l k}=\frac{-i}{2}\left(\delta_{i l} \delta_{j k}-\delta_{i k} \delta_{j l}\right) \quad(1 \leq i<j \leq n), \\
& M_{j}^{(3)}=\frac{\operatorname{diag}(1, \ldots, 1,-(j-1), 0, \ldots, 0)}{\sqrt{2 j(j-1)}} \quad(2 \leq j \leq n),
\end{aligned}
$$

where $M_{i j}^{(1)}, M_{i j}^{(2)}, M_{j}^{(3)}$ are the $n \times n$ dimensional generalization of the $S U(2)$ Pauli matrices $\sigma_{x, y, z}$ respectively. Counting the numbers of $S U(2 n)$ generators, there are $n(n-1) / 2$ real symmetric $M_{i j}^{1}$ 's, $n(n-1) / 2$ imaginary anti-symmetric $M_{i j}^{2}$ 's, and $n-1$ real diagonal $M_{i j}^{3}$ 's. Then the $S p(2 n)$ generators $X^{b}$ can be expressed as

$$
M_{i j}^{(2)} \otimes I_{2}, \quad M_{i j}^{(1)} \otimes \vec{\sigma}, \quad M_{j}^{(3)} \otimes \vec{\sigma}, \quad I_{n} \otimes \vec{\sigma},
$$

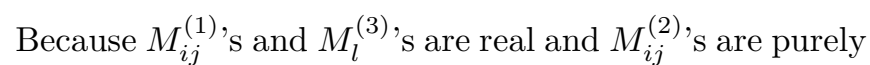
imaginary, the $S p(2 n)$ generators are odd under the time reversal: $T^{-1} X^{b} T=-X^{b}$. The second class bilinears $\psi_{\alpha}^{\dagger} Y_{\alpha \beta}^{a} \psi_{\beta}$ have $2 n^{2}-n-1$ elements. $Y^{a}\left(a=1, \ldots, 2 n^{2}-\right.$ $n-1)$ are given by

$$
M_{i j}^{(2)} \otimes \sigma_{i} \quad M_{j}^{(1)} \otimes I_{2}, \quad M_{j}^{(3)} \otimes I_{2},
$$

which are even under the time reversal: $T^{-1} Y^{a} T=Y^{a}$.

These $4 n^{2}$ bilinear operators are not independent of each other, but are related by the Fierz identity. The total Hilbert space for one site has the dimension of $2^{2 n}$, which can be decomposed into subspaces with different particle number $r(0 \leq r \leq 2 n)$. Each of them form the totally anti-symmetric representation of the $S U(2 n)$ group $1^{r}$. The Casimir value of the $S U(2 n)$ group in such representations are $r(2 n+1)(2 n-r) /(2 n)$. Thus we arrive the Fierz identity for the spin $n-\frac{1}{2}$ system as

$$
\begin{aligned}
& \sum_{b}\left(\psi_{i \alpha}^{\dagger} X_{\alpha \beta}^{b} \psi_{i \beta}\right)^{2}+\sum_{a}\left(\psi_{i \alpha}^{\dagger} Y_{\alpha \beta}^{a} \psi_{i \beta}\right)^{2} \\
+ & \frac{2 n+1}{2 n}\left(\psi_{\alpha}^{\dagger} \psi_{\alpha}-n\right)^{2}=\frac{2 n^{2}+n}{2} .
\end{aligned}
$$

The onsite pairing operators can be easily formed by using the $R$ matrix. Due to the Pauli's exclusion principle, the total spin for a s-wave pair can only be $0,2, \ldots, 2 n-2$. The singlet pair operator is also the $S p(2 n)$ singlet operator. It can be written as $\psi_{\alpha}^{\dagger} R_{\alpha \beta} \psi_{\beta}^{\dagger}$, which was studied extensively in a $S p(2 n)$ generalization of the Heisenberg antiferromagnet 32]. The other $2 n^{2}-n-1$ pairing operators with total spin $2,4, \ldots, 2 n-2$ together form a representation of $S p(2 n)$ as $\psi_{\alpha}^{\dagger}(i)\left(R Y^{a}\right)_{\alpha \beta} \psi_{\beta}^{\dagger}(i)$. When all the interaction parameters are equal, these $n(2 n-1)$ pairing operators together form anti-symmetric representation of $S U(2 n)$ of $1^{r}(\mathrm{r}=2)$.
[1] C. J. Wu, J. P. Hu, and S. C. Zhang, Phys. Rev. Lett. 91, 186402 (2003).

[2] R. Blankenbecler, D. J. Scalapino, and R. L. Sugar, Phys. Rev. D 24, 2278 (1981).

[3] J. E. Hirsch, Phys. Rev. B 31, 4403 (1985).

[4] S. Chandrasekharan and U. J. Wiese, Phys. Rev. Lett. 83, 3116 (1999).

[5] S. E. Koonin, D. J. Dean, and K. Langanke, Phys. Rep. 278, 1 (1997).

[6] S. Rombouts, K. Heyde, and N. Jachowicz, Phys. Rev. C 58, 3295 (1998).

[7] M. Imada and T. Kashima, J. Phys. Soc. Jpn. 69, 2723 (2000).

[8] T. Kashima and M. Imada, J. Phys. Soc. Jpn. 70, 2287 (2001).

[9] F. F. Assaad, V. Rousseau, F. Hebert, M. Feldbacher, and G. G. Batrouni, Europhysics Letters 63, 569 (2003).

[10] S. Capponi, C. J. Wu, and S. C. Zhang, Phys. Rev. B 70, 220505(R) (2004).

[11] D. Scalapino, S. C. Zhang, and W. Hanke, Phys. Rev. B 58, 443 (1998).

[12] J. E. Hirsch, Phys. Rev. B 28, 4059 (1983).
[13] Y. Q. Li, M. Ma, D. N. Shi, and F. C. Zhang, Phys. Rev. Lett. 81, 3527 (1998).

[14] E. Demler, W. Hanke, and S. C. Zhang, Rev. Mod. Phys. 76, 909 (2004).

[15] S. Murakami, N. Nagaosa, and S. C. Zhang, Phys. Rev. B 69, 235206 (2004).

[16] Y. Motome and M. Imada, J. Phys. Soc. Jpn. 66, 1872 (1997).

[17] T. L. Ho and S. Yip, Phys. Rev. Lett. 82, 247 (1999).

[18] P. Bouwknegt and K. Schoutens, Phys. Rev. Lett. 82, 2757 (1999).

[19] D. Duffy, S. Haas, and E. Kim, Phys. Rev. B 58, R5932 (1998).

[20] H. H. Lin, L. Balents, and M. P. A. Fisher, Phys. Rev. B 58, 1794 (1998).

[21] H. Frahm and M. Stahlsmeier, Phys. Rev. B 63, 125109 (2001).

[22] S. Chakravarty, R. B. Laughlin, D. K. Morr, and C. Nayak, Phys. Rev. B 63, 94503 (2001).

[23] C. Nayak, Phys. Rev. B 62, 4880 (2000).

[24] W. V. Liu and F. Wilczek, cond-mat/0312685 (2004).

[25] C. J. Wu, W. V. Liu, and E. Fradkin, Phys. Rev. B 68, 
115104 (2003).

[26] M. Tsuchiizu and A. Furusaki, Phys. Rev. B 66, 245106 (2002).

[27] C. J. Wu and S. C. Zhang, Phys. Rev. Lett. 93, 36403 (2004).

[28] J. E. Hirsch, Phys. Rev. B 42, 4774 (1990).

[29] J. E. Hirsch, Phys. Rev. B 41, 6820 (1990).
[30] S. Hands, I. Montvay, S. E. Morrison, M. Oevers, L. Scorzato, and J. Skellurd, Eur. Phys. J. C 17, 285 (2000).

[31] M. Hamermesh, Group Theory and Its Application to Physical Problems (Dover Pubns, 1989).

[32] S. Sachdev and Z. Wang, Phys. Rev. B 43, 10229 (1991). 